\title{
Emotional Context Distorts Both Time and Space in Children
}

\author{
Julie Fonseca Cruz ${ }^{1,2}$, Kévin Vidaud-Laperrière ${ }^{1}$, Claire Brechet ${ }^{1}$, Pom Charras ${ }^{1}$ \\ ${ }^{1}$ EA 4556 EPSYLON, Univ Paul Valéry Montpellier 3, Univercity Montpellier, Montpellier, France \\ ${ }^{2} \mathrm{CHU}$ Montpellier, University of Montpellier, Saint Eloi Hospital, Child and Adolescent Psychiatry Unit (MPEA1), Montpellier, \\ France \\ Email: ${ }^{*}$ pom.charras@univ-montp3.fr
}

How to cite this paper: Fonseca Cruz, J. Vidaud-Laperrière, K., Brechet, C. and Charras, P. (2020) Emotional Context Distorts Both Time and Space in Children. Journal of Behavioral and Brain Science, 10, 371-385.

https://doi.org/10.4236/jbbs.2020.109023

Received: July 13, 2020

Accepted: September 27, 2020

Published: September 30, 2020

Copyright $\odot 2020$ by author(s) and Scientific Research Publishing Inc. This work is licensed under the Creative Commons Attribution International License (CC BY 4.0).

http://creativecommons.org/licenses/by/4.0/

\begin{abstract}
According to a generalized magnitude system, the representation of time, space and number relies on a common cognitive mechanism. However, in the context of negative emotional stimuli, temporal durations undergo a subjective overestimation, while numerosity judgments are underestimated. This finding clearly challenged the existence of a generalized magnitude system. In this study, we aimed to investigate whether angry faces biases both temporal and spatial estimates compared to neutral faces in children aged 5 6-year-old and 9 - 10-year-old. Children were to judge as short or long either the temporal interval or the distance separating two visual stimuli in a bisection task. Overall, the study suggests that negative emotion with high arousal (angry faces) leads to a distortion of both duration and distance. Such distortion is reported early in development, even before the maturation of time perception.
\end{abstract}

\section{Keywords}

Emotion, Time, Space, Children, AToM

\section{Introduction}

High-arousal and negative stimuli are known to bias time perception resulting in a temporal lengthening effect. Interestingly, there is a controversy in the literature regarding whether this effect is specific to time perception or affects other dimensions such as space or number. While some studies suggest that magnitude estimations are differentially biased by high-arousal and negative emotional context [1] [2] [3], another study provided evidence for an analogue effect of emotion on time, space and numerosity [4]. Our objective in the present study is 
to investigate the impact of high-arousal and negative emotional context on both temporal and spatial estimations in children aged 5 to 10 .

In recent decades, ample evidence has highlighted developmental, functional and anatomical similarities in the perception and representation of magnitudes. In 2003, Walsh developed the AToM theory (A Theory of Magnitudes) which states the existence of a generalized magnitude system involving the inferior parietal cortex [5]. Magnitudes such as time, numerosity or space might share a common cognitive mechanism responsible for elaborating amodal representations of "more than-less than", "faster-slower", "bigger-smaller" [5] [6] [7]. One of the main behavioural arguments in favour of a "generalized magnitude system" relies on the observation that magnitude estimation is characterized by a scalar property of variance. Indeed, the variability of estimation increases with absolute magnitude [8] [9]. Human beings are thus able to perceive magnitude so as to estimate, represent and manipulate them [10]. More interestingly, infants are already capable of discriminating numerosity, duration or spatial characteristics of a stimulus (at least for easy ratios), suggesting an early development of this ability [11]. Precision evidently improves with age, as indexed by a decrease in Weber ratio [12] [13] [14]. Weber ratios are used to define perceptual sensitivity since they reflect the ratio of the just noticeable variation to the initial stimuli intensity. In general, Weber ratios are obtained by dividing the just noticeable difference (JND) by the bisection point (point of subjective equality, PSE). Although the ability to discriminate temporal duration, numerosity or spatial extent shows similar developmental trajectories [8] [15] [16], it is noteworthy that time differs from other magnitudes, in its requirement for cognitive control. Resources in attention and working memory are necessary to process temporal information, which is intrinsically sequential. In addition, it has recently been suggested that in children, improvement in acuity for temporal estimations is determined by attentional capabilities and working memory [17] [18] [19] [20] [21]. Given that attentional capabilities and working memory are not fully mature in young children; spatial judgments are much more accurate as compared to temporal estimations [22].

While both adults and children can make precise judgments of temporal interval or spatial extent, task-irrelevant stimulus characteristics, such as emotion, bias magnitude estimations. Notably, in the context of emotional stimuli, temporal durations undergo a subjective lengthening resulting in an overestimation [23]. When judging durations of faces expressing emotion, such as anger, happiness or sadness, participants show a bias towards an overestimation as compared to neutral stimuli [24]. These results can be explained by the increasing arousal level, since negative emotional stimulus induces an overestimation of duration in high-arousing condition [25]. Indeed, temporal distortion is more robust for negative valence emotion [24] [26] [27] and for stimulating stimuli [28]. Facial expressions such as anger or fear lead to a lengthening effect in temporal perception in adults but also in children as young as 3 years of age [26] 
[29]. Interestingly, magnitude dilation caused by emotional stimuli seems to be specific to time. Several attempts to investigate the effect of emotion on numerosity have failed to trigger an overestimation of numerosity. Based on a generalized magnitude system, negative emotions are supposed to bias magnitude estimation in the same direction, regardless of whether magnitude is time-, spaceor numerosity-based. In sharp contrast with this prediction, several studies consistently found that emotional context leads to numerosity underestimation [1] [2] [3]. In these studies, participants were to judge the number of items presented in each trial. To manipulate emotional context, Young and Cordes [3] inserted an emotional face stimulus (either neutral, angry or happy) before the to-be-estimated array of dots; while Baker, Rodzon and Jordan [1] asked participants to judge the number of emotional face stimuli presented simultaneously in an array (female face expressing either anger, happiness or neutrality). In both studies, the presence of angry faces led adult participants to underestimate the number of items. The impact of emotional context on numerosity estimation is thus independent of whether an angry face is presented immediately prior to a numeric bisection judgment [3]. It is also independent of whether participants were to judge the number of angry faces [1] or the number of items in the context of threatening stimuli (i.e. spiders; [2]). Despite this apparent consensus in the literature, a study conducted by Droit-Volet and collaborators [4] provided clear evidence for an overestimation of number and space in aversive context (auditory beep). Crucially, in this study, the mode of presentation for numerical and spatial magnitude was either non-sequential or sequential. In non-sequential mode of presentation, all the items in a given trial were presented simultaneously; while in the sequential mode, numerical or spatial information was not given all at once. Item presentation was a dynamic display (i.e. growing lines). In the latter, estimations of numerosity and space require resources in attention and working memory, so as to match cognitive demands involved in any temporal task. The results showed that an aversive beep biased both numerosity and space judgments towards an overestimation, but only when information was presented sequentially (this study is further discussed in the General discussion). Indeed, information which is provided sequentially, involves an accumulation system, regardless of the type of magnitudes (number and space). High-arousal stimuli may accelerate the speed of information accumulation or may impede potential loss of information [4]. To the best of our knowledge, no additional study in the literature has investigated how emotional faces impact spatial judgments, and no study has ever explored this phenomenon in children.

In the current study, we thus aimed to investigate whether children's spatial as well as temporal judgements are biased in emotional context of anger. Temporal judgments are biased by emotional context from the age of 3 [26] [29], but are not mature until 8 or 9 years old [30] [31] [32], the link between emotion and spatial judgment has been explored in two groups of children aged 5 and 10. If magnitude representation is supported by a generalized magnitude system, spa- 
tial judgments in emotional context should be distorted in a similar way, as are temporal judgments. To test this hypothesis, 5- and 10-year-old children performed either a spatial or a temporal bisection task while the emotional context was manipulated through the use of an angry versus neutral face (see Figure 1). Anger is a negative and high-arousal emotion, likely to produce important distortions. Indeed, the overestimation of angry expressions is consistently more important than for other emotional expressions and previous studies showed that it leads to temporal overestimations in children as young as 3 years-old (e.g., [24]). Additionally, in line with the study of Droit-Volet [4], spatial information was delivered sequentially in order to match the demands for information accumulation inherent to the temporal task. As interferences in magnitude estimation are supposed to emerge in working memory [33], we hypothesized that, in our experimental design, emotional facial expressions should bias spatial and temporal judgments.

\section{Methods}

\subsection{Participants}

A total of eighty-five children (aged 5 - 6 or 9 - 10) participated in this experiment: 54 children were between 5 and 6 years old (mean age_5; 7, SD_0.29, 27 girls and 27 boys) and 31 children were between 9 and 10 years old (mean age_9; 6, SD_0.22, 20 girls and 11 boys). For each age group, half of the children performed a temporal bisection task (judgments of duration) while the other half completed a spatial bisection task (judgments of distance). Group assignment was counter-balanced across participants. Note however that as the tasks proved to be very difficult, 10 children did not complete the experiment and their data were not included in the study (6 young children did not understand the task instructions in the temporal condition). All children were enrolled in kindergarten and primary schools located in the south of France (vicinity of Montpellier, Herault), where they followed the normal academic program.

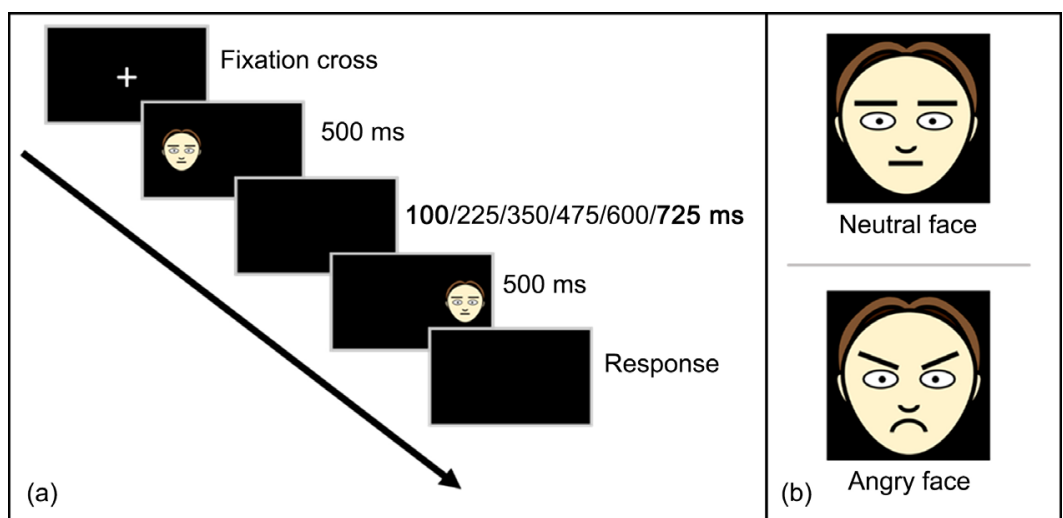

Figure 1. Panel (a) represents a schematic experimental trial design. Note that the distance between the two faces could be $4.98^{\circ}, 6.64^{\circ}, 8.3^{\circ}, 9.96^{\circ}, 11.62^{\circ}$ or $13.28^{\circ}$ during spatial task. Panel (b) represents the two facial expressions used (in a given trial, the two faces were either neutral or angry). 
The study, as well as the consent procedure for children, was approved by the "academy" (education authority) inspector of the French Ministry of National Education and by the children's teachers. The study was carried out according to the principles of the Helsinki Declaration and was approved by a local Research Ethics committee of University Paul Valery Montpellier. Written informed consent was obtained from the parents of all participants, as well as child assent.

\subsection{Stimuli and Procedure}

The experiment took place in a quiet room of the children's school where tables and chairs were adjusted to their size. Participants were invited to sit in front of a computer (17-inch screen) running E-Prime 2.0 software [34] to control stimuli presentation, timing operations and data collection. Verbal responses of participants were collected by the experimenter who reported the response by clicking on the left and right mouse buttons.

Stimuli were drawings of faces whose emotional facial expression was manipulated across trials. The faces represented either anger or neutral emotional facial expressions (see Figure 1, panel (b)). We used schematic faces instead of photographs because it has been shown that the use of such faces displaying only the basic facial components of emotional expressions (e.g., a downturned mouth and inward eyebrows) could help young children recognize the target emotion, particularly for anger and fear expressions [35]. The neutral and angry emotional status of schematic faces was equally presented through the experiment, in a random fashion.

Every trial started with a "fixation cross" centered on the screen (see Figure 1). After pressing the spacebar, a couple of faces subtended $1.74^{\circ}$ large $\times 3.1^{\circ}$ long were successively flashed on the screen for $500 \mathrm{~ms}$. The first face appeared either on the left or right of the fixation point and the second one was displayed on the opposite side so that the Left-to-Right/Right-to-Left direction of the faces was randomized between trials. The two faces were separated by a blank screen for a variable duration; between 100 and $725 \mathrm{~ms}$ ). Children gave verbal responses ("short" or "Iong") so that the experimenter reported the responses by pressing a button (left button for "short" and right button for "long" answer). Participants either performed a temporal or a spatial bisection task. In the temporal bisection task, participants were asked to judge as short or long the temporal interval between the first stimulus offset and the second stimulus onset. The actual duration lasted from 100 to $725 \mathrm{~ms}$ and could take 6 values (100/225/350/475/600/725 ms). In the spatial bisection task, participants were to estimate the distance separating the two faces as short or long. Faces were distant of $4.98^{\circ}, 6.64^{\circ}, 8.3^{\circ}, 9.96^{\circ}, 11.62^{\circ}$ and $13.28^{\circ}$. To match as much as possible the trial procedure for the two experimental tasks, the distance between faces varied between the shortest $\left(4.98^{\circ}\right)$ and the longest distance $\left(13.28^{\circ}\right)$ in the temporal task; and in the spatial task, the temporal interval between the faces was either short (100 ms) or long (725 ms). Participants first received instructions about 
the experimental tasks and were presented twice (one for each emotional facial expression-neutral and angry) with the short and long durations (100/725 ms) or distances $\left(4.98 / 13.28^{\circ}\right)$ depending on the task. In the second step, a training block familiarized participants with the two temporal anchors in the temporal bisection task $(100 / 725 \mathrm{~ms})$ or with the two spatial anchors in the spatial bisection task $\left(4.98 / 13.28^{\circ}\right)$. Participants performed 8 training trials (4 short and 4 long, in a randomized order). A response was considered correct when the participant responded "short" for the shortest duration/distance and "long" for the longest duration/distance. Conversely, a response was considered incorrect when the participant responded "short" for the longest intervals and "long" for the shortest intervals. For each response, children were provided with a feedback $(1000 \mathrm{~ms})$, following Droit-Volet and Wearden's procedure [31]. When giving a correct answer, the children saw the word "Correct" displayed on the screen alongside a clapping hands picture. In contrast, in case of an incorrect response, "Incorrect" appeared with a red exclamation mark. In the experimental block, participants were to judge whether the current duration or distance between the emotional faces was closer to the shortest or longest anchors previously learned. The procedure used in the experimental trials was identical to that of the training trials, except that children were not given a feedback anymore. Participants performed 12 trials per experimental condition, for a total of 144 estimations: 6 intervals (spatial or temporal) $\times 2$ emotions (neutral or angry) $\times 12$ repetitions.

\section{Statistical Analysis}

Given participants' age and task difficulty, data were trimmed to discard from statistical analyses responses from children who showed extremely low performance on the anchors (shortest duration or distance and longest duration or distance). We calculated for each participant the proportion of Long responses (thereafter $\mathrm{p}$ (long)) separately for the shortest distance or duration and the longest distance or duration (depending on the task). We expected $\mathrm{p}$ (long) to be close to 0 for the shortest anchor and close to 1 for the longest anchor. We then computed the difference between $\mathrm{p}$ (long) at short and long anchors. The higher the difference score, the better the performance. The data of children with a difference score below 0.3 were excluded from statistical analyses. Given that the task proved clearly difficult for many children, this led us to discard data from 10 additional participants (6 young children in the temporal bisection task). Consequently, the data of twenty-one 5 - 6-year-old children and twelve 9 10 -year-old children were analyzed for the temporal bisection task, and the data of sixteen 5 - 6-year-old children and sixteen 9 - 10-year-old children were included in the statistical analyses of the spatial bisection task. We performed a repeated measures ANOVA on the proportion of "long" responses with the factors "Task" (Spatial vs. Temporal), and "Age" (5 - 6 years old or 9 - 10 years old) manipulated between participants, and with the factors "Emotion" (Angry; Neutral), and "Magnitude" (Distance 1, 2, 3, 4, 5, 6 or Duration 1, 2, 3, 4, 5, 6) ma- 
nipulated within participants.

Then, to further explore the influence of emotional face stimuli on time and space perception, we computed for each participant and every experimental condition the bisection point (BP - also referred to as the Point of Subjective Equality, PSE). Each BP value was calculated from a slope and an intercept, obtained by fitting the psychometric raw data with the Pphy package on $\mathrm{R}$ [36]. Pphy is an R package that uses the model-free package to fit the data locally per subject, which were included as a random effect to account for the repeated-measures nature of the design as well as individual differences in response scale use. The slope, the bisection point and the JND (just noticeable difference) are extracted per subject and condition. BP represents the theoretical value in which the probability to judge a distance or duration as being long is 0.5. A low BP value refers to an overestimation and a high $\mathrm{BP}$ value to an underestimation. To assess perceptual sensitivity, Weber ratios (WRs) were calculated by dividing the JND with the BP for each participant and in every experimental condition. Additionally, as we aimed to compare perceptual distortion (accuracy of estimates) in the temporal and the spatial task, BPs were transformed into z-scores. Once standardized, the data in the temporal and spatial tasks could be statistically compared and the effect of Emotion in the two tasks and in the two age groups was thus explored. We first ran a repeated-measure ANOVA on WRs as a function of Emotion and Task, manipulated within subjects, and of Age manipulated between subjects. Then we ran a repeated-measure ANOVA on normalized BPs as a function of Emotion and Task, manipulated within subjects, and of Age manipulated between subjects.

\section{Results}

The ANOVA on the long responses (see Table 1) showed a main significant effect of Magnitude $\left(F(5,305)=340.26, p<0.001, \eta^{2} p=0.85\right)$, revealing that participants were able to correctly perform the tasks. It also revealed a main effect of the Task $\left(F(1,61)=7.65, p=0.007, \eta^{2} p=0.11\right)$, and a significant interaction between Magnitude $\times$ Task $\left(F(5,305)=7.35, p<0.001, \eta^{2} p=0.11\right)$ showing that, as expected, performance in the spatial task was higher than in the temporal one. The analysis showed no main significant effect of Age $(F(1,61)=2.53, p$ $\left.=0.12, \eta^{2} p=0.04\right)$, but a significant interaction between Age $\times$ Magnitude $(F(5$, $305)=7.52, p<0.001, \eta^{2} p=0.110$ ), suggesting that 9 - 10-year-old children performed better than 5 - 6-year-old participants especially on the longest durations and distances. The interaction between Age $\times$ Magnitude $\times$ Task $(F(5,305)=$ 3.03, $\left.p=0.01, \eta^{2} p=0.05\right)$ showed that the difference in performance between the two tasks (Spatial > Temporal) was higher for children aged 9 - 10 (see Figure 2: right panel) than for 5 - 6-year-old participants (see Figure 2: left panel).

Finally, with respect to the factor "Emotion", the analysis revealed a significant main effect $\left(F(1,61)=7.31, p=0.009, \eta^{2} p=0.11\right)$ revealing that angry face stimuli led children to provide less "long" responses. The effect of Emotion 
Table 1. Means and Standard Errors (SE) of the proportion of "long" responses as a function of Magnitude (distance or duration) and Task (spatial or temporal) for each age group.

\begin{tabular}{|c|c|c|c|c|c|c|c|c|}
\hline \multirow{2}{*}{ Age } & \multirow{2}{*}{ Task } & \multirow{2}{*}{$\mathrm{n}$} & Magnitude 1 & Magnitude 2 & Magnitude 3 & Magnitude 4 & Magnitude 5 & Magnitude 6 \\
\hline & & & Mean (SE) & Mean (SE) & Mean (SE) & Mean (SE) & Mean (SE) & Mean (SE) \\
\hline \multirow{2}{*}{5 years old } & Spatial Task & $(n=16)$ & $0.17(0.024)$ & $0.25(0.034)$ & $0.37(0.046)$ & $0.59(0.046)$ & $0.75(0.035)$ & $0.80(0.026)$ \\
\hline & Temporal Task & $(n=21)$ & $0.14(0.021)$ & $0.22(0.030)$ & $0.35(0.040)$ & $0.47(0.040)$ & $0.57(0.031)$ & $0.69(0.023)$ \\
\hline \multirow{2}{*}{9 years old } & Spatial Task & $(\mathrm{n}=16)$ & $0.04(0.024)$ & $0.12(0.034)$ & $0.39(0.046)$ & $0.67(0.046)$ & $0.89(0.035)$ & $0.97(0.026)$ \\
\hline & Temporal Task & $(\mathrm{n}=12)$ & $0.19(0.028)$ & $0.25(0.039)$ & $0.34(0.053)$ & $0.50(0.053)$ & $0.67(0.041)$ & $0.82(0.030)$ \\
\hline
\end{tabular}

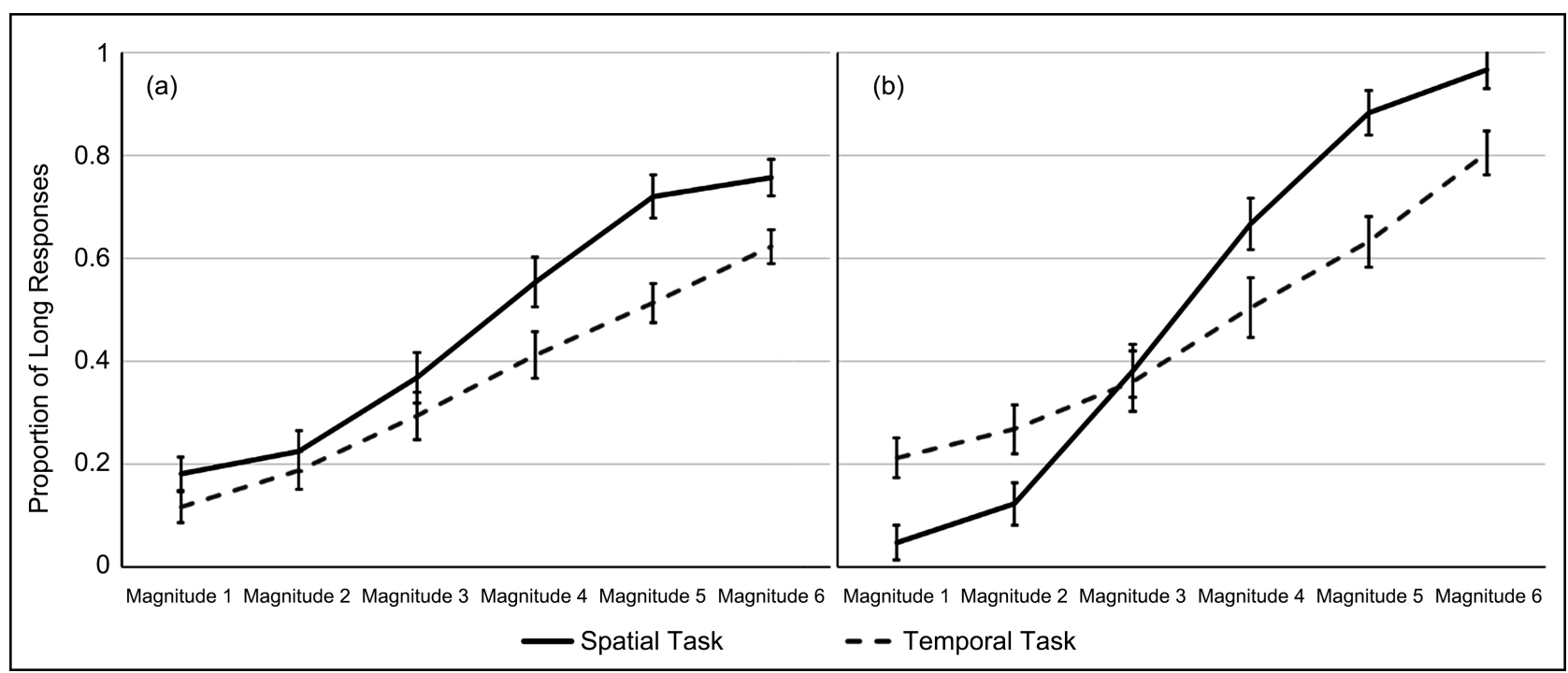

Figure 2. Shows the proportions of "long" responses as a function of Magnitude and Task separately for the 5 - 6-year-old participants (panel (a)) and the 9 - 10-year-old participants (panel (b)).

showed that both temporal and spatial judgments were underestimated when faces were angry as opposed to neutral (Figure 3 ). As expected, the interaction between Emotion $\times$ Magnitude was also significant $(F(5,305)=3.26, p=0.007$, $\left.\eta^{2} p=0.05\right)$, suggesting that the effect of "Emotion" is higher on intermediate magnitudes (distances 2, 3, 4 and 5, or durations 2, 3, 4 and 5) than on extreme magnitudes. But importantly, the interaction between Task $\times$ Emotion $\times$ Magnitude was not significant $\left(F<1, p=0.47, \eta^{2} p=0.01\right)$, revealing that Emotion modulates temporal and spatial estimates to the same extent. All the remaining interactions did not reach the threshold significance (Task $\times$ Age, $F<1, p=0.51$, $\mathrm{ns}$; Emotion $\times$ Age, $F(1,61)=1.25, p=0.27$, ns; Emotion $\times$ Task, $F(1,61)=1.7, p$ $=0.20$, ns; Emotion $\times$ Task $\times$ Age, $F<1, p=0.66$, ns; Emotion $\times$ Age $\times$ Magnitude, $F<1, p=0.55$, ns; Task $\times$ Emotion $\times$ Age $\times$ Magnitude, $F<1, p=0.48$, ns).

The ANOVA on WRs revealed a main effect of Task $(F(1,61)=42.09, p<$ $\left.0.001, \eta^{2} p=0.41\right)$, since WRs are much larger in the temporal than the spatial task. The results also showed a significant interaction between Task $\times$ Age $(F(1$, $\left.61)=5.99, p=0.02, \eta^{2} p=0.09\right)$, suggesting that the difference in WRs between 
the spatial and temporal task is more pronounced for children aged 10 (see Table 2). The statistical analysis thus confirmed that sensitivity is higher for children aged 10 in the spatial task.

For the ANOVA on BPs, as data were standardized for each participant in the two tasks and as a function of Age, we only focused on the main effect and interactions involving the factor Emotion. The main effect of Emotion was highly significant $\left(F(1,61)=8.47, p=0.005, \eta^{2} p=0.12\right)$, confirming the statistical analysis on $\mathrm{p}$ (long). It suggests that children underestimated both temporal intervals and covered distances when presented with angry faces in opposition to neutral faces. The interactions involving the factor Emotion did not reach significance (Emotion $\times$ Age, $F(1,61)=1.88, p<0.17, \eta^{2} p=0.03$; Emotion $\times$ Task, $(F$ $<1, p=0.42, \mathrm{~ns})$. Emotion $\times$ Age $\times$ Task, $(F<1, p=0.89, \mathrm{~ns}))$.

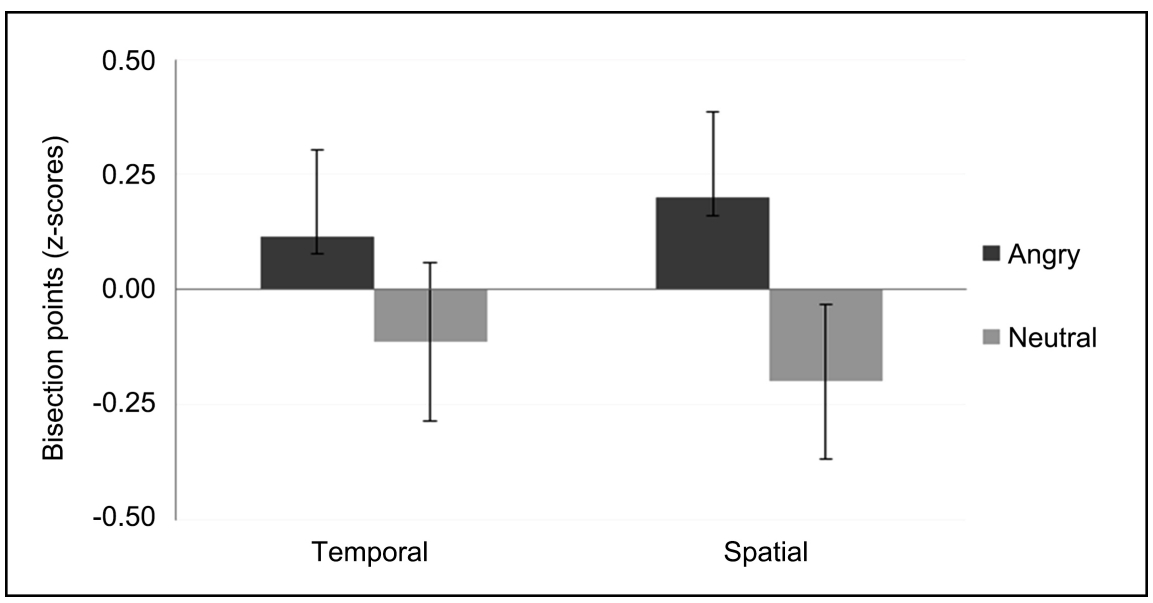

Figure 3. Shows the normalized bisection points as a function of Emotion (Angry and Neutral) for all children and separately for the temporal and spatial tasks. Note that positive values refer to an underestimation, and negative values to an overestimation.

Table 2. Means and Standard Errors (SE) of the Bisection points (BP in ms or mm), WRs and normalized BP as a function of Emotion (Angry and Neutral) and Task (spatial or temporal) for each age group.

\begin{tabular}{|c|c|c|c|c|c|c|}
\hline \multirow{2}{*}{ Age } & \multirow{2}{*}{ Task } & \multirow{2}{*}{$\mathrm{n}$} & \multirow{2}{*}{ Emotion } & \multirow{2}{*}{$\begin{array}{c}\text { Bisection Point } \\
\text { Mean (SE) }\end{array}$} & \multirow{2}{*}{$\begin{array}{c}\text { WR } \\
\text { Mean (SE) }\end{array}$} & \multirow{2}{*}{$\begin{array}{c}\text { Normalized BP } \\
\text { Mean (SE) }\end{array}$} \\
\hline & & & & & & \\
\hline \multirow{6}{*}{5 years ol } & \multirow{4}{*}{ Spatial Task } & \multirow{3}{*}{$(\mathrm{n}=16)$} & Angry & $949(31.23)$ & $0.25(0.043)$ & $0.134(0.26)$ \\
\hline & & & & & & \\
\hline & & & Neutral & $916(28.16)$ & $0.28(0.042)$ & $-0.134(0.24)$ \\
\hline & & \multirow{3}{*}{$(\mathrm{n}=21)$} & & & & \\
\hline & \multirow[b]{2}{*}{ Temporal Task } & & Angry & $510(27.27)$ & $0.41(0.038)$ & $0.032(0.23)$ \\
\hline & & & Neutral & $501(24.58)$ & $0.44(0.037)$ & $-0.032(0.21)$ \\
\hline \multirow{7}{*}{9 years o } & \multirow{3}{*}{ Spatial Task } & \multirow{3}{*}{$(n=16)$} & Angry & $931(31.23)$ & $0.13(0.043)$ & $0.267(0.26)$ \\
\hline & & & & & & \\
\hline & & & Neutral & $873(28.16)$ & $0.14(0.042)$ & $-0.267(0.24)$ \\
\hline & \multirow{4}{*}{ Temporal Task } & & & & & \\
\hline & & \multirow{3}{*}{$(\mathrm{n}=12)$} & Angry & $479(36.06)$ & $0.47(0.043)$ & $0.195(0.30)$ \\
\hline & & & & & & \\
\hline & & & Neutral & $444(32.51)$ & $0.47(0.048)$ & $-0.195(0.27)$ \\
\hline
\end{tabular}




\section{Discussion}

In the current study, children were to judge either the duration or the distance of an empty interval between two faces. The aim of the study was to investigate the effect of Emotion on magnitude estimation. A large body of evidence suggests that, in adults and children, temporal estimates are biased towards dilation in an emotional context of anger or fear [26]. However, little is known about the effect of Emotion on spatial judgments, and even less in children. The results on $\mathrm{p}$ (long) and WRs indicate that even if children aged 5 and 9 were able to correctly perform the tasks, they were more precise in the spatial task, as compared to the temporal task. Casasanto et al. [22] already reported that children are more successful in a spatial discrimination task than in a temporal discrimination task. These observations are consistent with the literature, notably with the results of McCormak et al. [37] that showed that accurate estimates of duration improved until adulthood (for review see [17]).

The results on the BP show the first piece of evidence for distorted magnitude judgments of both time and space in children, caused by emotional faces. Children from 5 to 10 underestimated temporal intervals and covered distances when demarcated by angry faces. The presence of angry faces thus distorted magnitude estimates in the same way and to a similar extent as regards both temporal and spatial judgments. Previous studies showed that durations were overestimated in the context of threatening stimuli [30] [38] [39] while we observed the opposite effect for both temporal and spatial judgments. Indeed, children underestimated not only durations but also distances when presented with an angry face compared to a neutral one. Moreover, this "shortening effect" was found irrespective of age in both children aged 5 to 6 or 9 to 10 (as revealed by BPs).

This opposite effect of emotion on quantity estimation was surprising but has already been reported in the literature on time estimation [40] [41]. In accordance with the theory of Ono and collaborators, this magnitude underestimation can be explained by the experimental procedure used in our bisection task. Participants in a standard temporal bisection task are asked to estimate the duration of visually (or auditory) presented stimuli. However, in the present study, children were to estimate an empty interval between two stimuli, instead of stimulus duration per se. Ono \& Kitazawa [40] developed the "retrospective shortening hypothesis" to account for the shortening effect, according to which the increasing arousal caused by the to-be-timed event decreases the subjective duration of the preceding event (see also [42]). Consequently, we hypothesize that participants in our study oriented their attention toward faces, particularly toward angry faces, and neglected information between these items. When facial expression evoked anger, information between the two faces was even more neglected, resulting in an underestimation of not only temporal intervals but also covered distances.

The shortening effect for duration and distance can also be explained by the expectancy effect. When individuals focus their attention on a highly expected 
event, their attention is diverted away from elapsed time. This effect is usually observed in dual-task paradigms [43] [44]. For instance, Droit-Volet et al. [44] showed that children aged 5 and 8 underestimate time when dual tasked because allocation of attentional resources interferes with temporal judgments. In our study, the procedure required children to wait until the second stimulus before responding. One can therefore hypothesize that children turn their attention toward the arrival of the second stimulus, thus triggering an expectancy effect. In "angry" trials, given the high level of arousal provoked by negative emotion, the expectancy effect is possibly enhanced. Children might process less efficiently magnitude information and therefore perceive both duration and distance as being shorter.

Our results are in complete agreement with a couple of studies of Droit-Volet in which the authors provided evidence for a common effect of emotion on time [45], numerosity and length perception [4]. In these studies, adult participants were to assess duration, numerosity or line length after being exposed to an aversive auditory stimulus. Numerical and spatial magnitudes were presented sequentially and non-sequentially. The results were clear-cut in the sense that during sequential presentation, numerosity and length estimations were biased towards an overestimation in the context of an aversive noise, just like temporal estimations. The present study extends these findings to children, and shows that the mechanisms underlying distortions in magnitude estimates are already mature at age 5 . These results are also in line with a recent study of Charras et al. [42] in which they provide evidence that temporal estimates in 5-year old children are biased by irrelevant spatial dimension in the same way and to the same extent as adults.

Yet, note that our findings seem contradictory with recent research work providing evidence for differential effects of emotion on quantities. As mentioned in the Introduction, a series of studies have consistently reported that emotion differentially affects numerosity and time. Numerical estimations are biased towards an underestimation in the context of negative emotion [1] [2] [3]. As argued by the authors, this finding clearly runs counter to a generalized magnitude system. Nonetheless, in the three aforementioned studies (see also [46]), the timing task and the numerosity task did not only differ in the magnitude at hand, but also involved different demands in terms of information updating or accumulation. Timing tasks were much more demanding than numerosity tasks with regard to working memory requirements. To time an event, one has to remember the onset of an event and accumulate temporal information so as to estimate duration. In sharp contrast, for numerosity and line length, information can be provided all at once at a given moment. When information is presented simultaneously, there is no need for magnitude accumulation, neither for demands on working memory (see [47]). Controlling for sequential presentation is essential to compare magnitude estimation in time and in other dimensions [9]. In the present study, since the two faces were never presented at the same time 
but were instead successively flashed, information accumulation was required for both spatial and temporal judgments. As a consequence, we assume that the influence of emotional context (in particular high-arousal and negative) occurs at a memory stage during the accumulation process. Distortions of magnitude estimates, either temporal or spatial, might emerge during information updating for both adults and children. This assumption is entirely consistent with recent studies suggesting that interferences between magnitudes (leading to perceptual distortions) arise at a working memory stage [33].

\section{Conclusions}

Overall, the present study confirms the results of Droit-Volet et al. [4] [38] and does extend the findings to children from 5 years old. When individuals make magnitude judgments in which information accumulation is required, a negative emotion with high arousal leads to a distortion of both duration and distance. Such distortion is reliable early in development, even before the maturation of time perception. Our findings are in accordance with the idea that magnitudes might share common resources and cognitive system and that the accumulation process involved in temporal or spatial estimation is a weak point where distortions may emerge.

\section{Conflicts of Interest}

The authors declare no conflicts of interest regarding the publication of this paper.

\section{References}

[1] Baker, J.M., Rodzon, K. and Jordan, K. (2013) The Impact of Emotion on Numerosity Estimation. Frontiers in Psychology, 4, 521. https://doi.org/10.3389/fpsyg.2013.00521

[2] Hamamouche, K.A., Niemi, L. and Cordes, S. (2017) Quantifying a Threat: Evidence of a Numeric Processing Bias. Acta Psychologica, 177, 1-9. https://doi.org/10.1016/j.actpsy.2017.04.001

[3] Young, L.N. and Cordes, S. (2013) Fewer Things, Lasting Longer: The Effects of Emotion on Quantity Judgments. Psychological Science, 24, 1057-1059. https://doi.org/10.1177/0956797612465294

[4] Droit-Volet, S. (2013) Emotion and Magnitude Perception: Number and Length Bisection. Frontiers in Neurorobotics, 7, 24. https://doi.org/10.3389/fnbot.2013.00024

[5] Walsh, V. (2003) A Theory of Magnitude: Common Cortical Metrics of Time, Space and Quantity. Trends in Cognitive Sciences, 7, 483-488. https://doi.org/10.1016/j.tics.2003.09.002

[6] Bueti, D. and Walsh, V. (2009) The Parietal Cortex and the Representation of Time, Space, Number and Other Magnitudes. Philosophical Transactions of the Royal Society of London B: Biological Sciences, 364, 1831-1840. https://doi.org/10.1098/rstb.2009.0028

[7] Dormal, V. and Pesenti, M. (2009) Common and Specific Contributions of the 
Intraparietal Sulci to Numerosity and Length Processing. Human Brain Mapping, 30, 2466-2476. https://doi.org/10.1002/hbm.20677

[8] Droit-Volet, S., Clément, A. and Fayol, M. (2008) Time, Number and Length: Similarities and Differences in Discrimination in Adults and Children. Quarterly Journal of Experimental Psychology, 61, 1827-1846. https://doi.org/10.1080/17470210701743643

[9] Droit-Volet, S. (2010) Speeding Up a Master Clock Common to Time, Number and Length? Behavioural Processes, 85, 126-134. https://doi.org/10.1016/j.beproc.2010.06.017

[10] Crollen, V., Grade, S., Pesenti, M. and Dormal, V. (2013) A Common Metric Magnitude System for the Perception and Production of Numerosity, Length, and Duration. Frontiers in Psychology, 4, 449. https://doi.org/10.3389/fpsyg.2013.00449

[11] de Hevia, M.D., Izard, V., Coubart, A., Spelke, E.S. and Streri, A. (2014) Representations of Space, Time, and Number in Neonates. Proceedings of the National Academy of Sciences, 111, 4809-4813. https://doi.org/10.1073/pnas.1323628111

[12] Brannon, E.M. (2006) The Representation of Numerical Magnitude. Current Opinion in Neurobiology, 16, 222-229. https://doi.org/10.1016/j.conb.2006.03.002

[13] Halberda, J. and Feigenson, L. (2008) Developmental Change in the Acuity of the "Number Sense": The Approximate Number System in 3-, 4-, 5-, and 6-Year-Olds and Adults. Developmental Psychology, 44, 1457. https://doi.org/10.1037/a0012682

[14] Odic, D., Libertus, M.E., Feigenson, L. and Halberda, J. (2013) Developmental Change in the Acuity of Approximate Number and Area Representations. Developmental Psychology, 49, 1103. https://doi.org/10.1037/a0029472

[15] Brannon, E.M., Suanda, S. and Libertus, K. (2007) Temporal Discrimination Increases in Precision over Development and Parallels the Development of Numerosity Discrimination. Developmental Science, 10, 770-777. https://doi.org/10.1111/j.1467-7687.2007.00635.x

[16] Feigenson, L. (2007) The Equality of Quantity. Trends in Cognitive Sciences, 11, 185-187. https://doi.org/10.1016/j.tics.2007.01.006

[17] Droit-Volet, S. (2016) Development of Time. Current Opinion in Behavioral Sciences, 8, 102-109. https://doi.org/10.1016/j.cobeha.2016.02.003

[18] Droit-Volet, S., Wearden, J. and Zélanti, P.S. (2015) Cognitive Abilities Required in Time Judgment Depending on the Temporal Tasks Used: A Comparison of Children and Adults. Quarterly Journal of Experimental Psychology, 68, 2216-2242. https://doi.org/10.1080/17470218.2015.1012087

[19] Hallez, Q. and Droit-Volet, S. (2017) High Levels of Time Contraction in Young Children in Dual Tasks Are Related to Their Limited Attention Capacities. Journal of Experimental Child Psychology, 161, 148-160. https://doi.org/10.1016/j.jecp.2017.04.013

[20] Hallez, Q. and Droit-Volet, S. (2019) Timing in a Dual-Task in Children and Adults: When the Interference Effect Is Higher with Concurrent Non-Temporal than Temporal Information. Journal of Cognitive Psychology, 31, 34-48. https://doi.org/10.1080/20445911.2019.1567519

[21] Zélanti, P.S. and Droit-Volet, S. (2011) Cognitive Abilities Explaining Age-Related Changes in Time Perception of Short and Long Durations. Journal of Experimental Child Psychology, 109, 143-157. https://doi.org/10.1016/j.jecp.2011.01.003

[22] Casasanto, D., Fotakopoulou, O. and Boroditsky, L. (2010) Space and Time in the Child's Mind: Evidence for a Cross-Dimensional Asymmetry. Cognitive Science, 34, 
387-405. https://doi.org/10.1111/j.1551-6709.2010.01094.x

[23] Droit-Volet, S. and Meck, W.H. (2007) How Emotions Colour Our Perception of Time. Trends in Cognitive Sciences, 11, 504-513.

https://doi.org/10.1016/j.tics.2007.09.008

[24] Droit-Volet, S., Brunot, S. and Niedenthal, P. (2004) Perception of the Duration of Emotional Events. Cognition and Emotion, 18, 849-858. https://doi.org/10.1080/02699930341000194

[25] Angrilli, A., Cherubini, P., Pavese, A. and Manfredini, S. (1997) The Influence of Affective Factors on Time Perception. Attention, Perception and Psychophysics, 59, 972-982. https://doi.org/10.3758/BF03205512

[26] Gil, S. and Droit-Volet, S. (2011) How Do Emotional Facial Expressions Influence our Perception of Time? In: Masmoudi, S., Yan Dai, D. and Naceur, A., Eds., Attention, Representation, and Human Performance: Integration of Cognition, Emotion and Motivation, Psychology Press, Taylor \& Francis, London, 61-74.

[27] Tipples, J. (2008) Negative Emotionality Influences the Effects of Emotion on Time Perception. Emotion, 8, 127. https://doi.org/10.1037/1528-3542.8.1.127

[28] Fayolle, S.L. and Droit-Volet, S. (2014) Time Perception and Dynamics of Facial Expressions of Emotions. PLoS ONE, 9, e97944. https://doi.org/10.1371/journal.pone.0097944

[29] Gil, S., Niedenthal, P.M. and Droit-Volet, S. (2007) Anger and Time Perception in Children. Emotion, 7, 219. https://doi.org/10.1037/1528-3542.7.1.219

[30] Droit-Volet, S., Tourret, S. and Wearden, J. (2004) Perception of the Duration of Auditory and Visual Stimuli in Children and Adults. Quarterly Journal of Experimental Psychology Section A, 57, 797-818.

https://doi.org/10.1080/02724980343000495

[31] Droit-Volet, S. and Wearden, J. (2001) Temporal Bisection in Children. Journal of Experimental Child Psychology, 80, 142-159. https://doi.org/10.1006/jecp.2001.2631

[32] Droit-Volet, S. and Wearden, J. (2002) Speeding Up an Internal Clock in Children? Effects of Visual Flicker on Subjective Duration. Quarterly Journal of Experimental Psychology Section B, 55, 193-211. https://doi.org/10.1080/02724990143000252

[33] Cai, Z.G., Wang, R., Shen, M. and Speekenbrink, M. (2018) Cross-Dimensional Magnitude Interactions Arise from Memory Interference. Cognitive Psychology, 106, 21-42. https://doi.org/10.1016/j.cogpsych.2018.08.001

[34] Schneider, W., Eschman, A. and Zuccolotto, A. (2002) E-Prime User's Guide. Psychology Software Tools Inc., Pittsburgh.

[35] Brechet, C. (2017) Children's Recognition of Emotional Facial Expressions through Photographs and Drawings. The Journal of Genetic Psychology: Research and Theory on Human Development, 178, 139-146. https://doi.org/10.1080/00221325.2017.1286630

[36] R Core Team (2014) R: A Language and Environment for Statistical Computing. R Foundation for Statistical Computing, Vienna. http://www.R-project.org

[37] McCormack, T., Brown, G.D., Maylor, E.A., Darby, R.J. and Green, D. (1999) Developmental Changes in Time Estimation: Comparing Childhood and Old Age. Developmental Psychology, 35, 1143. https://doi.org/10.1037/0012-1649.35.4.1143

[38] Droit-Volet, S., Fayolle, S., Lamotte, M. and Gil, S. (2013) Time, Emotion and the Embodiment of Timing. Timing \& Time Perception, 1, 99-126. https://doi.org/10.1163/22134468-00002004

[39] Droit-Volet, S., Fayolle, S.L. and Gil, S. (2011) Emotion and Time Perception: Ef- 
fects of Film-Induced Mood. Frontiers in Integrative Neuroscience, 5, 33. https://doi.org/10.3389/fnint.2011.00033

[40] Ono, F. and Kitazawa, S. (2010) The Effect of Perceived Motion-in-Depth on Time Perception. Cognition, 115, 140-146.

https://doi.org/10.1016/j.cognition.2009.12.006

[41] Ono, F. and Watanabe, K. (2011) Attention Can Retrospectively Distort Visual Space. Psychological Science, 22, 472-477. https://doi.org/10.1177/0956797611403319

[42] Charras, P., Droit-Volet, S., Brechet, C. and Coull, J.T. (2017) The Spatial Representation of Time Can Be Flexibly Oriented in the Frontal or Lateral Planes from an Early Age. Journal of Experimental Psychology: Human Perception and Performance, 43, 832. https://doi.org/10.1037/xhp0000349

[43] Coull, J.T., Vidal, F., Nazarian, B. and Macar, F. (2004) Functional Anatomy of the Attentional Modulation of Time Estimation. Science, 303, 1506-1508. https://doi.org/10.1126/science.1091573

[44] Droit-Volet, S., Provasi, J., Delgado, M. and Clément, A. (2005) Le développement des capacités de jugement des durées chez l'enfant. Psychologie Française, 50, 145-166. https://doi.org/10.1016/j.psfr.2004.10.007

[45] Droit-Volet, S., Mermillod, M., Cocenas-Silva, R. and Gil, S. (2010) The Effect of Expectancy of a Threatening Event on Time Perception in Human Adults. Emotion, 10, 908. https://doi.org/10.1037/a0020258

[46] Lewis, E.A., Zax, A. and Cordes, S. (2018) The Impact of Emotion on Numerical Estimation: A Developmental Perspective. Quarterly Journal of Experimental Psychology, 71, 1300-1311. https://doi.org/10.1080/17470218.2017.1318154

[47] Coull, J.T., Charras, P., Donadieu, M., Droit-Volet, S. and Vidal, F. (2015) SMA Selectively Codes the Active Accumulation of Temporal, Not Spatial, Magnitude. Journal of Cognitive Neuroscience, 27, 2281-2298.

https://doi.org/10.1162/jocn a 00854 